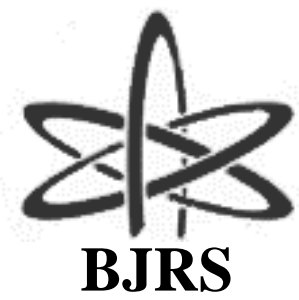
BRAZILIAN JOURNAL
$\mathrm{OF}$
RADIATION SCIENCES
06-01 (2018) 01-13

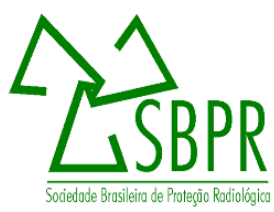

\title{
Avaliação da dose e do contraste em sistemas de mamografia computadorizada - CR
}

\author{
C. D. Almeida ${ }^{a}$; L. T. L. Sardo $;$ J. E. Peixoto ${ }^{\text {c. }}$ \\ ${ }^{a}$ Instituto de Radioproteção e Dosimetria, CEP 22783-127, Rio de Janeiro-RJ, Brasil claudio@ird.gov.br \\ ${ }^{\mathrm{b}}$ Universidade Federal do Rio de Janeiro, CEP 21941-909, Rio de Janeiro-RJ, Brasil. \\ ${ }^{c}$ Instituto Nacional de Câncer José Alencar Gomes da Silva (INCA), CEP 20231-092, Rio de Janeiro, RJ, Brasil.
}

\begin{abstract}
RESUMO
Novas tecnologias em radiologia digital são uma realidade no país e avançam substituindo os sistemas convencionais. Em mamografia, o sistema digital mais usado é a radiografia computadorizada (CR). Este sistema, quando não otimizado, pode requerer o emprego de doses no tecido glandular da mama acima dos níveis aceitáveis para se obter imagens com contraste adequado. A otimização dos sistemas de mamografia digital em um serviço tem como referência os valores da razão contraste-ruído (CNR) e da dose glandular média (DGM) usados pelo sistema de imagem (mamógrafo e digitalizador CR). Este estudo foi realizado em um serviço que utiliza três mamógrafos e dois digitalizadores CR de diferentes fabricantes, formando cinco sistemas de imagem distintos. Ele teve como objetivo comparar o desempenho destes sistemas em termos das CNRs para as técnicas radiográficas ( $\mathrm{kV}$, combinação alvo/filtro e mAs) utilizadas para radiografar mamas de diferentes espessuras e das respectivas DGMs. Os resultados mostram que, nos cinco sistemas, as técnicas radiográficas usadas para radiografar mamas de diferentes espessuras produziam DGMs dentro dos níveis aceitáveis, porém, as CNRs estavam inferiores aos valores aceitáveis, indicando que o serviço estava produzindo imagens com contraste reduzido ou ruído aumentado. Foi constatado que a otimização da CNR e da DGM não foi implementada devido a limitações do dispositivo de controle automático de exposição (CAE). Assim, o processo de otimização possível deverá estabelecer as técnicas radiográficas que produzem as maiores CNRs com as DGMs não ultrapassando os níveis aceitáveis para as mamas de diferentes espessuras.
\end{abstract}

Palavras-chave: CNR, Dose glandular, Mamografia digital. 


\begin{abstract}
New technologies in digital radiology are a reality in Brazil and they are replacing the screen/film system. In mammography, the preferable system is the computerized radiology, CR. This system, when not optimized, could deliver glandular tissue doses above the acceptable ones to obtain images with adequate contrast. The optimization of the mammography digital systems in a facility uses as references the values of the the contrast to noise ratio, CNR, and the mean glandular dose, MGD, used by the image system (mammography equipment and CR digitizer). This study was performed in a facility that uses three mammography equipment and two CR digitizers from different manufactures, totalizing five different image systems. Aiming to compare the performance of these systems in terms of the CNRs values for the radiography techniques $(\mathrm{kV}$, target/filter combination and $\mathrm{mAs})$ used to acquire images from various breast thickness, and their respective MGDs. The results show that, at the five systems, the radiography techniques used to acquire breast images from distinctive thickness, was delivering acceptable values of MGD, however, the CNR values were below the acceptable ones, demonstrating that the facility was producing images with reduced contrast or increased noise. It was verified that the optimization of the CNR and MGD was not implemented due to the limitations of the Automatic Exposure Control, AEC. Therefore, the possible optimization process should establish the radiography techniques that produces superior values of CNR, not exceeding the MGD acceptable values for different breast thickness.
\end{abstract}

Keywords: CNR, glandular dose, digital mammography.

\title{
1. INTRODUÇÃO
}

A transição da mamografia com o sistema filme-tela intensificadora para a mamografia digital está progredindo rapidamente no Brasil, com a predominância dos sistemas de mamografia computadorizada (CR). O uso das novas tecnologias vem contribuindo cada vez mais para melhorar o diagnóstico em mamografia. Entretanto, elas trazem grandes desafios no estabelecimento dos padrões de desempenho dos equipamentos, dos critérios de qualidade das imagens clínicas e na otimização das doses de radiação recebidas pelas pacientes [1], [2]. Resultados do estudo realizado por Coutinho [3] mostram a tendência de doses acima dos níveis aceitáveis em serviços que usam o sistema CR. 
A otimização do contraste de imagem em mamografia permite maximizar o seu benefício, por meio de uma melhor precisão do diagnóstico. Além disso, a otimização da dose recebida pela paciente permite manter sob controle o risco de câncer radioinduzido.

A avaliação dos padrões de desempenho dos parâmetros de operação do mamógrafo e do sistema de detecção da imagem é uma rotina do programa de controle de qualidade (PCQ) do serviço. Entretando, os níveis de tolerância dos parâmetros avaliados no PCQ que constam da Portaria SVS/MS N 453 de 01/06/1998 se referem apenas ao sistema filme-tela intensificadora [4]. Parâmetros de desempenho dos sistemas digitais, tais como: razão contraste-ruído (CNR) e linearidade da resposta do detector têm seus níveis de tolerância estabelecidos na publicação "Quality Assurance Programme for Digital Mammography" da Agencia Internacional de Energia Atômica [5], a partir de agora denominada IAEA HHS No 17 . Nesta publicação, também estão estabelecidos os níveis aceitáveis da dose glandular média (DGM) para mamas de diferentes espessuras.

A identificação de imagens representativas de lesões com características de malignidade depende do seu contraste e do nível de ruído presente na região em que se encontram [6]. O parâmetro que descreve a relação entre o contraste e o ruído em uma imagem clínica é a CNR e, por conseguinte, ele deve ser otimizado [7], mantendo a DGM igual ou abaixo dos níveis aceitáveis [5].

Este estudo teve como objetivo comparar as doses glandulares médias recebidas pelas pacientes e o contraste da imagem para as técnicas radiográficas utilizadas $(\mathrm{kV}$, combinação alvo/filtro e mAs) para radiografar mamas de diferentes espessuras em um serviço de mamografia que utiliza três mamógrafos e dois digitalizadores CR de diferentes fabricantes, formando cinco sistemas de imagem distintos.

\section{MATERIAIS E MÉTODOS}

Este estudo foi realizado em um serviço que possui três mamógrafos convencionais das marcas GE modelo Senographe DMR, Lorad modelo M-IV e Philips modelo Graph Mammo. Estes mamógrafos operam com dois sistemas de radiografia computadorizada (CR), das marcas Kodak 
modelo Direct View Elite e Agfa modelo CR 85-X com as respectivas placas de imagem (IP) modelos EHR-M2 e MM 3.0.

A placa de imagem EHR-M2 tem tamanho $18 \mathrm{~cm}$ x $24 \mathrm{~cm}$ e a imagem é gravada em 16 bits/pixel em uma matriz de $3584 \times 4784$ pixeis de tamanho 48,5 $\mu \mathrm{m}$ e tempo de uso de 18 meses. A placa MM 3.0 tem tamanho $18 \mathrm{~cm}$ x $24 \mathrm{~cm}$ e a imagem é gravada em 16 bits/pixel em uma matriz de $3560 \times 4640$ pixeis de tamanho $50 \mu \mathrm{m}$ e tempo de uso de 6 meses.

Foi usado o detector Unfors modelo 535L nas medidas do rendimento dos mamógrafos e no teste de linearidade de resposta do detector. Para medir a razão contraste-ruído (CNR), foram usadas placas de polimetilmetacrilato (PMMA), lamina de alumínio de $10 \mathrm{~mm}^{2}$ de área e $0,2 \mathrm{~mm}$ de espessura e réguas de PVC para serem usadas como espaçadores.

Os mamógrafos GE e Lorad foram ajustados para trabalhar com os sistemas CR da Kodak e da Agfa. Já o mamógrafo Philips foi ajustado somente para trabalhar com o sistema Agfa. A Tabela 1 mostra os conjuntos de sistemas avaliados.

Tabela 1. Marca e modelo dos mamógrafos e dos digitalizadores CR e modelo das placas de imagem dos sistemas avaliados.

\begin{tabular}{cccc}
\hline Sistema & $\begin{array}{c}\text { Mamógrafo } \\
\text { (marca / modelo) }\end{array}$ & $\begin{array}{c}\text { Digitalizador } \\
\text { (marca / modelo) }\end{array}$ & $\begin{array}{c}\text { Placa de } \\
\text { Imagem (IP) }\end{array}$ \\
\hline $\mathbf{1}$ & Philips / Graph Mammo & Agfa / CR 85-X & MM 3.0 \\
$\mathbf{2}$ & GE / DMR & Agfa / CR 85-X & MM 3.0 \\
$\mathbf{3}$ & Lorad / M-IV & Agfa / CR 85-X & MM 3.0 \\
$\mathbf{4}$ & Lorad / M-IV & Kodak/ Direct View & EHR M2 \\
$\mathbf{5}$ & GE / DMR & Kodak / Direct View & EHR M2 \\
\hline
\end{tabular}

O mamógrafo GE tem as opções de combinação alvo/filtro Mo/Mo, Mo/Rh e Rh/Rh e os mamógrafos Lorad e Philips têm as opções Mo/Mo e Mo/Rh. Estes sistemas foram avaliados em relação à linearidade de resposta do detector, dose glandular média e razão contraste-ruído. A Figura 1a mostra o arranjo experimental para medir o rendimento do tubo e posteriormente calcular a DGM e a Figura 1b mostra o arranjo para realizar as medidas da CNR. 
Figura 1: Arranjo experimental para medir o rendimento do tubo (a) e para medir a razão contraste-ruído (b).

(a)

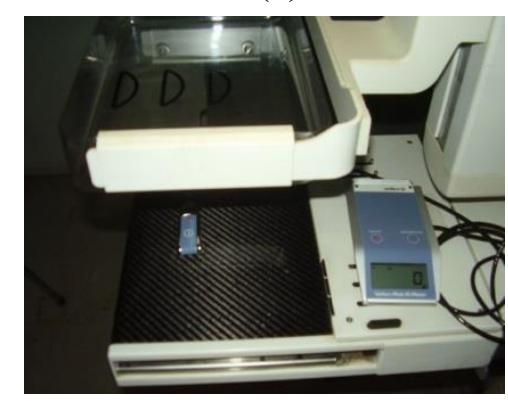

(b)

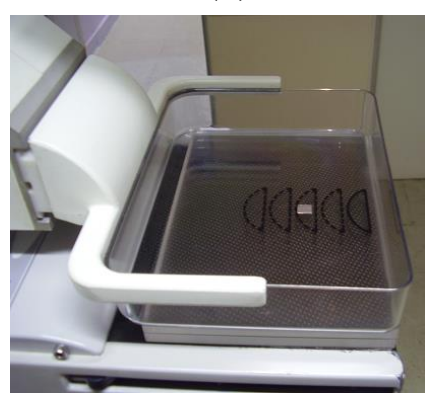

Para a medida da linearidade de resposta do detector em cada um dos cinco sistemas foi posicionada uma placa de PMMA com espessura de $45 \mathrm{~mm}$ de PMMA na saída do feixe de raios-X do mamógrafo. Para cada sistema mamógrafo-digitalizador $\mathrm{CR}$, foi feita uma série de nove imagens usando sempre a mesma placa de imagem posicionada sobre o bucky com exposições de $28 \mathrm{kV}$, combinação alvo/filtro Mo/Mo e valores do produto da corrente $x$ tempo de exposição que variaram de $4 \mathrm{mAs}$ a $450 \mathrm{mAs}$ [5]. A seguir, a série de nove exposições foi repetida para a medida do kerma no ar de entrada $\left(K_{i}\right)$ na posição da placa de imagem. Após as leituras das imagens pelo digitalizador $\mathrm{CR}$, foram medidos os valores médios de pixel $(p)$ e o desvio padrão do valor de pixel $\left(\sigma_{p}\right)$ em uma região de interesse $(\mathrm{ROI})$ de $10 \mathrm{~mm}^{2}$ de área posicionada a $6 \mathrm{~cm}$ da borda da parede torácica e centrada lateralmente. Para estas imagens foi determinada a relação entre os valores de $p$ e os $K_{i}$ respectivos [5]. Ela foi usada para linearizar os valores de $p$ e os respectivos desvios padrões dos valores do pixel nas ROIs de cada imagem $\left(\sigma_{p}\right)$ em função de $K_{i}$. Os valores de $\sigma_{p}$ nas ROIs de cada imagem foram usados para investigar a relação entre o ruído na imagem $\left(\sigma_{p}\right)$ e o kerma no dectector, representado por $p$ linearizado. A relação entre o ruído relativo $\left(\sigma_{p}\right.$ $/ p$ ) linearizado e o kerma no detector foi determinada empiricamente por meio de um ajuste de potência, mostrado na Equação 1 [7].

$$
\frac{\sigma_{p}}{p}=k_{t} \cdot p^{-n}
$$


onde $k_{t}$ é uma constante. Quando o ruído na imagem é puramente de natureza quântica, o valor de $n$ deve ser 0,5 . Se houver ruído de natureza eletrônica ou devido às estruturas materiais do detector, $n$ pode ser superior ou inferior a 0,5 .

Os valores da DGM e da CNR foram determinados para sete espessuras diferentes de mama comprimida, conforme procedimentos descritos na publicação European protocol for the quality control of the physical and technical aspects of mammography screening [8]. Para isso, foram usadas placas de PMMA variando de $20 \mathrm{~mm}$ a $70 \mathrm{~mm}$ de espessura que correspondem a mamas comprimidas de $21 \mathrm{~mm}$ a $90 \mathrm{~mm}$. As placas foram posicionadas sobre o bucky e sobre a placa com 20 mm de PMMA foi colocada uma lâmina de alumínio com $10 \mathrm{~mm}^{2}$ e 0,2 mm de espessura centrada lateralmente e a $6 \mathrm{~cm}$ da borda da parede torácica. As imagens foram obtidas usando o dispositivo de controle automático de exposição (CAE) do mamógrafo e sempre a mesma placa de imagem. As técnicas radiográficas (kV/alvo/filtro e mAs) selecionadas pelo CAE para cada espessura foram usadas para a determinação de $K_{i}$. Os valores médios de pixel $(p)$ os desvios padrões do valor de pixel $\left(\sigma_{p}\right)$ foram obtidos em todas as imagens com o uso do software ImageJ. Os valores de CNR foram calculados para todas as espessuras utilizando a Equação 2 [7].

$$
C N R=\frac{p-p(A l)}{\sqrt{\frac{\sigma^{2}+\sigma(A l)^{2}}{2}}}
$$

Onde: $p$ e $p(A l)$ são os valores médios de pixel linearizados nas regiões fora e dentro da imagem da placa de alumínio e $\sigma_{p}$ e $\sigma(A l)$ são os desvios padrões linearizados dos valores de pixel fora e dentro da imagem da placa de alumínio.

Para determinar a DGM, primeiro foi necessário medir o rendimento do mamógrafo para todas as combinações kV/alvo/filtro obtidas com o uso do CAE. Com estes valores de rendimento, foi calculado o valor do $K_{\mathrm{i}}$ para cada espessura de mama comprimida. O cálculo da DGM foi feito utilizando a equação 3 [5].

$$
D G M=K_{i} \cdot g . c . s
$$


onde os fatores $g$ e c dependem de valores da glandularidade da mama, da camada semi-redutora do mamógrafo e da espessura da mama, e o fator $s$ dependem da combinação alvo/filtro utilizada. Estes fatores são encontrados na literatura [9]. As camadas semi-redutoras para todas as qualidades do feixe utilizadas foram medidas em todos os mamógrafos.

\section{RESULTADOS E DISCUSSÃO}

A Tabela 2 mostra os coeficientes de correlação linear $\left(\mathrm{R}^{2}\right)$ entre o valor médio de pixel $(p)$ linearizado e kerma no ar incidente $\left(K_{\mathrm{i}}\right)$, o ruído relativo $\left(\sigma_{p} / p\right)$ e o índice de potência $(n)$ da Equação 1, para os diferentes sistemas de imagem estabelecidos com a combinação $\mathrm{kV} /$ alvo/filtro de 28 $\mathrm{kV} / \mathrm{Mo} / \mathrm{Mo}$ para os cinco sistemas de imagem avaliados neste trabalho.

Tabela 2. Coeficiente de correlação linear $\left(\mathrm{R}^{2}\right)$ entre o valor médio de pixel $(p)$ linearizado e kerma no ar incidente $\left(K_{\mathrm{i}}\right)$, ruído relativo $\left(\sigma_{p} / p\right)$ e índice de potência $(n)$ da Equação 1, para os diferentes sistemas de imagem.

\begin{tabular}{cccc}
\hline SISTEMA & $\mathbf{R}^{\mathbf{2}}$ & $\boldsymbol{\sigma}_{p} / \boldsymbol{p}$ & $\boldsymbol{n}$ \\
\hline $\mathbf{1}$ & 0,998 & 0,59 & 0,41 \\
$\mathbf{2}$ & 0,999 & 0,62 & 0,38 \\
$\mathbf{3}$ & 1,000 & 0,61 & 0,39 \\
$\mathbf{4}$ & 1,000 & 0,57 & 0,43 \\
$\mathbf{5}$ & 0,999 & 0,61 & 0,39 \\
\hline
\end{tabular}

Os resultados da resposta do detector em termos de $p$ linearizado como uma função de $K_{\mathrm{i}}$ indicam que os cinco sistemas podem ser considerados lineares tendo em vista que apresentaram valores do quadrado do coeficiente de correlação $\left(\mathrm{R}^{2}\right)$ acima de 0,99 [5].

A variação do ruído em função do $K_{\mathrm{i}}$ foi analisada plotando o desvio padrão do valor de pixel linearizado para cada valor de $K_{\mathrm{i}}$ como mostrado nas Figuras $2 \mathrm{a}-\mathrm{e}$. 
Figura 2: Desvio padrão do valor médio do pixel em função do kerma no ar de entrada na superfície do detector dos cinco sistemas testados.

(a)

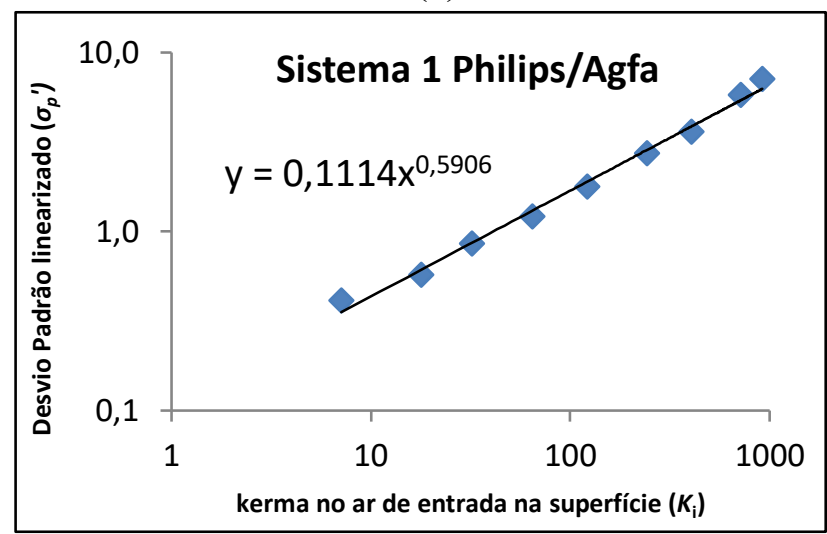

(c)

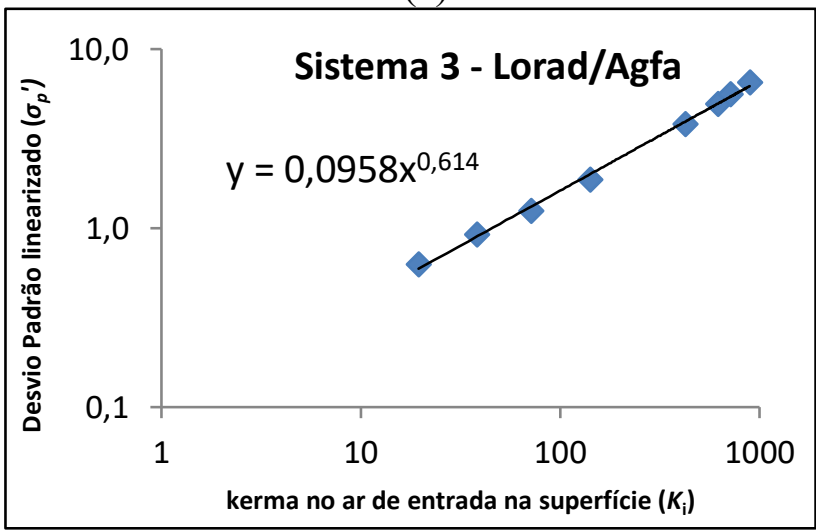

(b)

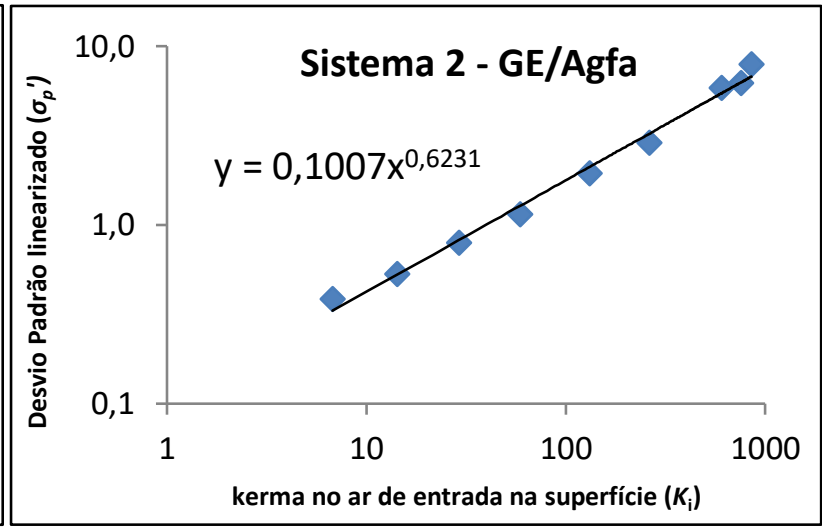

(d)

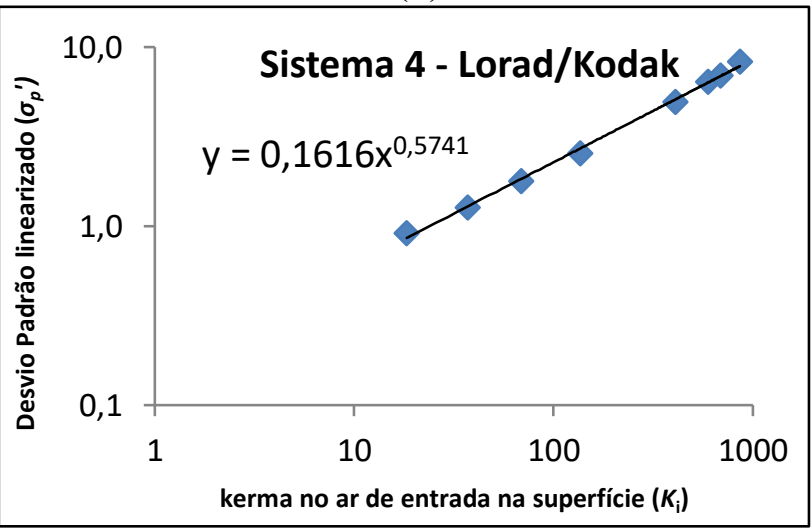

(e)

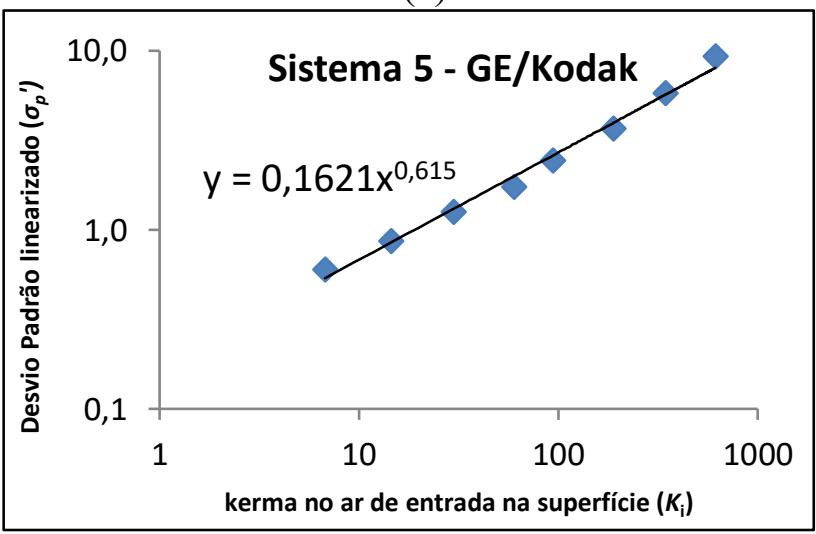


Se o ruído de natureza quântica fosse o único presente, os dados resultariam em uma linha reta com índice $n$ de 0,5. A presença de ruído estrutural deu origem a um desvio da linha reta. Esta é uma situação normal para este tipo de tecnologia de sistema de imagem. Pode ser observado na Tabela 2 e nas Figuras 3a-e que os valores de $n$ mostrado nos gráfico variaram de 0,38 a 0,43, o que sugere a presença de quantidades variáveis de ruído devido às estruturas materiais das placas de imagem. As Figuras 3a-e apresentam uma forma alternativa de representar os mesmos dados mostrando o ruído relativo para os diferentes valores médios de pixel.

Figura 3: Ruído relativo em função do valor médio do pixel linearizado nos cinco sistemas testados.

(a)

(b)

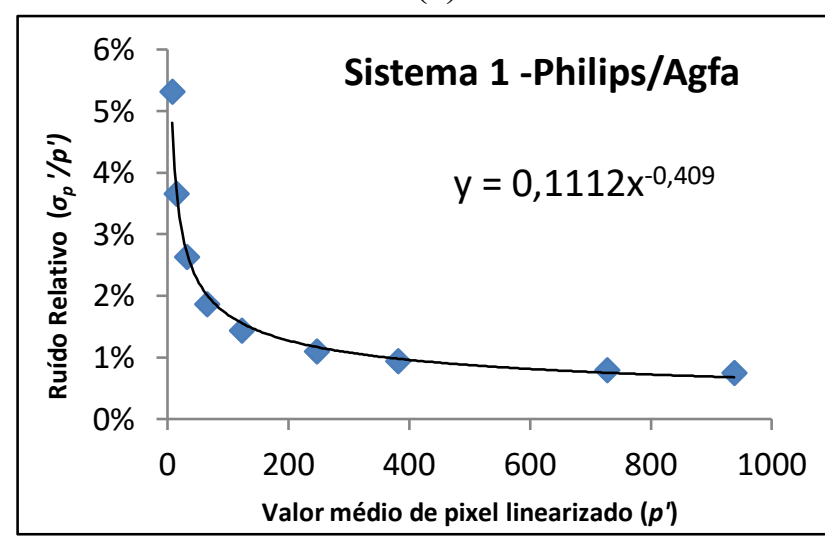

(c)

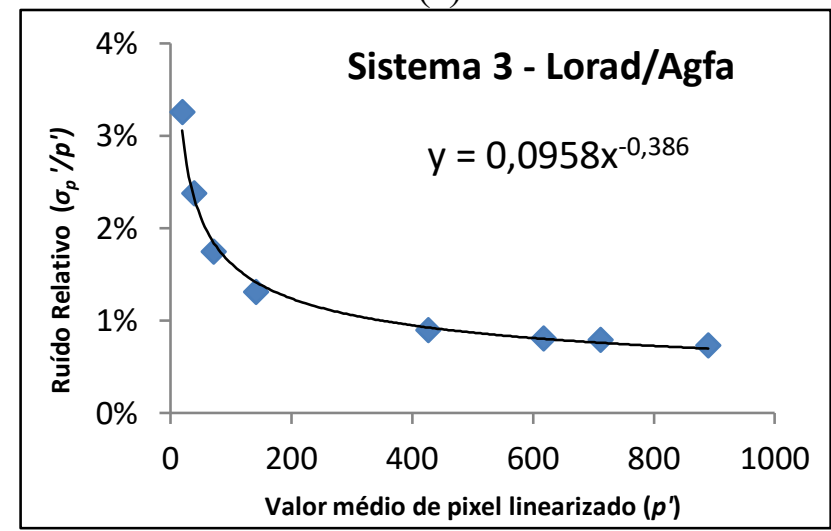

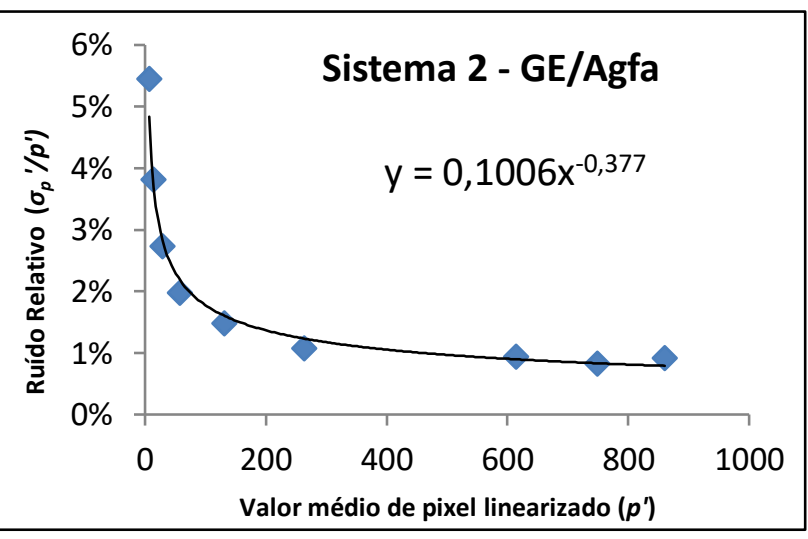

(d)

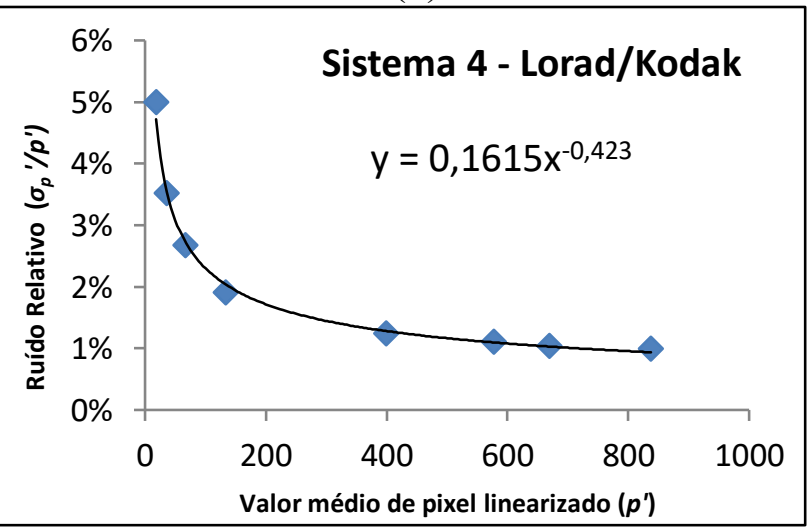


(e)

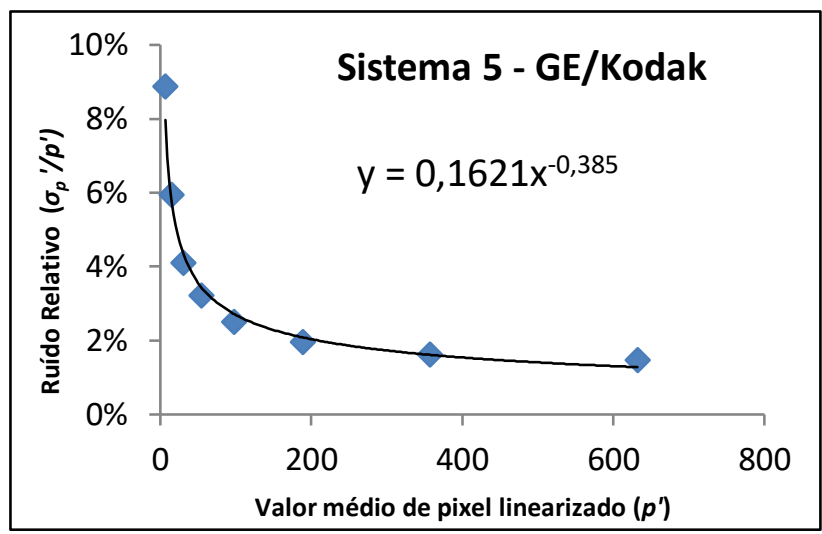

Os valores das razões contraste-ruído obtidos para diferentes espessuras de mama simuladas por placas de PMMA são mostrados na Figura 4a-b. Na Figura 4a pode ser observado que, para os três sistemas com digitalizador Agfa, a CNR medida estava abaixo do valor mínimo aceitável para mamas de espessura igual ou acima de $45 \mathrm{~mm}$. O mesmo ocorreu com os dois sistemas com o digitalizador Kodak, como mostra a Figura 4b. Neste caso, a CNR só estava acima do mínimo aceitável no sistema 4, Lorad/Kodak, para a espessura de $21 \mathrm{~mm}$. Estes resultados indicam a necessidade de ajuste em todos os sistemas para garantir o contraste adequado da imagem.

Figura 4: Valores medidos e mínimo aceitável da razão contraste-ruído (CNR) (a) Sistemas 1 - Philips/Agfa, 2 - GE/Agfa, e 3 - Lorad/Agfa, (b) Sistemas 4 - Lorad/Kodak e 5 - GE/Kodak.

(a)

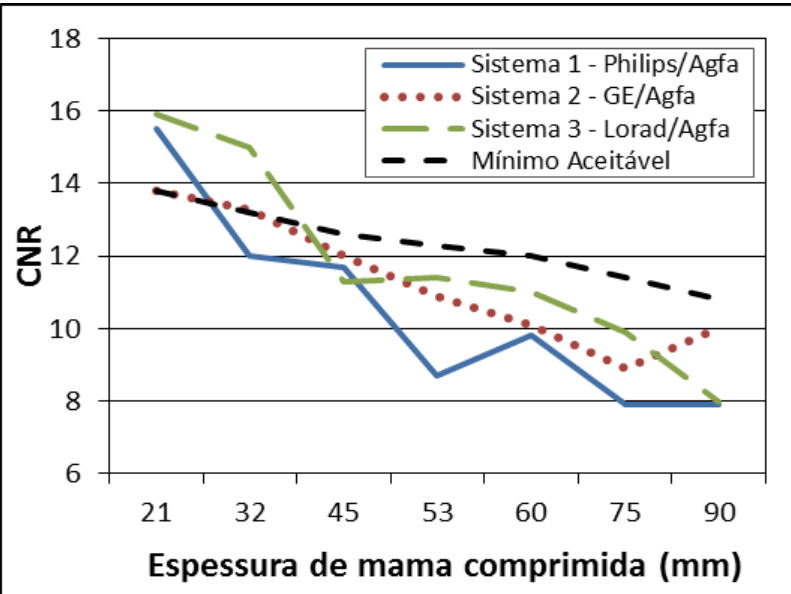

(b)

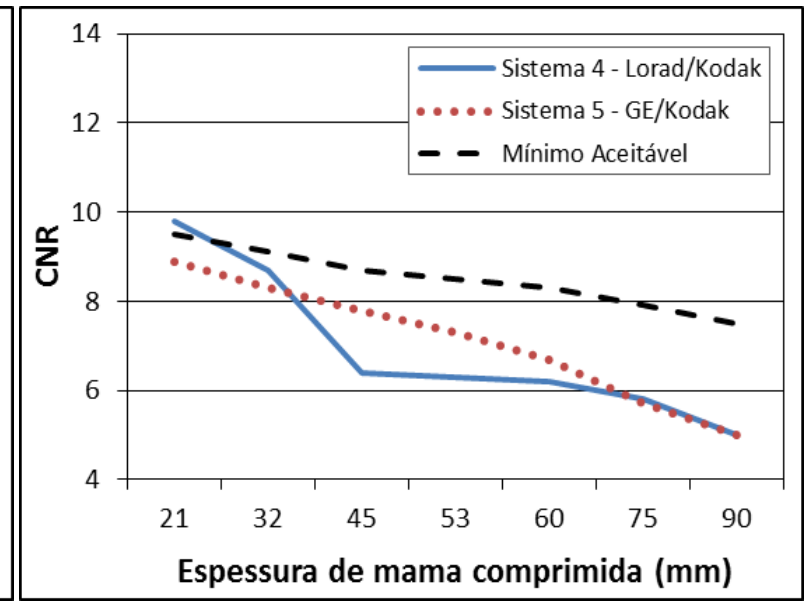


A Tabela 3 mostra os valores de DGM medidas nos sistemas testados para as várias espessuras equivalentes a mama comprimida e os valores aceitáveis apresentados pela publicação IAEA HHS No 17 [5].

Em todos os sistemas as medidas de DGM ficaram igual ou abaixo dos valores aceitáveis. Entretanto, como mostrado na Figura 4a-b, os resultados das CNRs estão inferiores ao mínimo aceitável em todos os sistemas para mamas de espessuras acima de $32 \mathrm{~mm}$ devido à pouca quantidade de radiação que atinge as placas de imagem. Estes resultados indicam que o dispositivo de controle automático de exposição (CAE) dos mamógrafos não alcança um equilíbrio para a DGM em toda a faixa de espessura de mama comprimida, gerando assim, valores de CNR inferiores aos necessários, principalmente nas mamas mais espessas.

Tabela 3. Dose glandular média (DGM) medida nos cinco sistemas de imagem e valor aceitável pela publicação IAEA HHS $\mathrm{N}^{\mathbf{0}} 17$ [5] para cada espessura equivalente de mama comprimida.

\begin{tabular}{|c|c|c|c|c|c|c|c|}
\hline & & \multicolumn{5}{|c|}{ SISTEMAS } & \multirow{3}{*}{$\begin{array}{c}\text { DGM aceitável } \\
\text { (mGy) }\end{array}$} \\
\hline \multicolumn{2}{|c|}{ Espessura (mm) } & 1 & 2 & 3 & 4 & 5 & \\
\hline PMMA & MAMA & \multicolumn{5}{|c|}{ DGM medida (mGy) } & \\
\hline 20 & 21 & 0,9 & 0,6 & 0,8 & 0,6 & 0,6 & $<1,0$ \\
\hline 30 & 32 & 1,4 & 1,0 & 1,4 & 1,0 & 1,2 & $<1,5$ \\
\hline 40 & 45 & 2,0 & 1,8 & 1,4 & 0,8 & 1,9 & $<2,0$ \\
\hline 45 & 53 & 1,9 & 2,0 & 1,8 & 1,1 & 2,3 & $<2,5$ \\
\hline 50 & 60 & 2,5 & 2,3 & 2,4 & 1,6 & 2,8 & $<3,0$ \\
\hline 60 & 75 & 4,1 & 3,4 & 3,6 & 2,6 & 3,8 & $<4,5$ \\
\hline 70 & 90 & 5,4 & 6,5 & 4,5 & 3,5 & 4,4 & $<6,5$ \\
\hline
\end{tabular}

Em todos os sistemas testados, ficou evidente a necessidade de que as técnicas radiográficas sejam otimizadas de modo que o ruído na imagem seja reduzido por meio de aumento da DGM, observando que ela não ultrapasse os valores aceitáveis. Para tal, pode ser empregada a metodologia apresentada por Almeida [7]. Ela se aplica à otimização das técnicas radiográficas para os sistemas digitais utilizando o índice de potência ( $n)$, CNR e DGM. 


\section{CONCLUSÕES}

Os resultados mostram que, nos cinco sistemas, as DGMs para mamas de todas as espessuras estavam iguais ou abaixo do nível aceitável. Entretanto, as CNRs estavam inferiores aos valores aceitáveis, indicando que o serviço estava produzindo imagens com contraste reduzido ou ruído aumentado.

Foi constatado que a otimização da CNR e da DGM não foi implementada devido às limitações do dispositivo do controle automático de exposição (CAE). Assim, o processo de otimização possível deverá estabelecer as técnicas radiográficas que produzem as maiores CNRs com as DGMs não ultrapassando os níveis aceitáveis para as mamas de diferentes espessuras.

Embora os sistemas de radiologia computadorizada, $\mathrm{CR}$, requeiram valores de doses mais elevados, quando comparados com os sistemas filme-tela intensificadora e de mamografia digital direta (DR) para alcançar contraste adequado e evitar ruído na imagem, ainda assim devem ser submetidos a um processo otimização.

\section{REFERÊNCIAS}

1. Freitas AG, Kemp C, Louveira MH, Fujiwara SM e Campos LF. Mamografia digital: perspectiva atual e aplicações futuras. Radiologia Brasileira 2006; 39 (4): 287-296.

2. Obenauer S, Hermann KP and Grabbe E. Dose reduction in full-field digital mammography: an anthropomorphic breast phantom study. The British Journal of Radiology 2003; 76:478-482.

3. Coutinho CMC. Avaliação da dose glandular em sistemas de mamografia convencional e digital utilizando um fantoma dosimétrico, [tese - Doutorado]. Rio de Janeiro: COPPE, Universidade Federal do Rio de Janeiro, 2009.

4. Ministério da Saúde, Diretrizes básicas de proteção radiológica em radiodiagnóstico médico e odontológico. Portaria 453, 01 de junho de 1998, Brasil.

5. IAEA Human Health Series No. 17, Quality Assurance Programme for Digital Mammography, IAEA 2011. Disponível em: http://www-pub.iaea.org/MTCD/ publications/PDF/Pub1482_ web.pdf [consultado em 30/09/2014]. 
6. Chevalier M. La Física de la Mamografía, Curso Mamografía Digital: fundamentos y control de calidad. Madrid: Sociedad Española de Física Médica, 2012:18-21.

7. Almeida, C.D., Otimização da dose glandular média na mama e da qualidade da imagem nos sistemas de mamografia digital, [tese - Doutorado]. Rio de Janeiro, HUCFF, Universidade Federal do Rio de Janeiro, 2014.

8. Van Engen R, Young KC, Bosmans H and Thijssen M. European protocol for the quality control of the physical and technical aspects of mammography screening. Part B: Digital Mammography. In European Guidelines for Breast Cancer Screening, Fourth edition. European Commission 2006.

9. Dance DR, Skinner CL, Young KC, Beckett JR and Kotre CJ. Additional factors for the estimation of mean glandular breast dose using the UK mammography dosimetry protocol. Phys. Med. Biol. 2000;45:3225-3240. 